\title{
Inteligencia y Memoria de Trabajo: La Relación Entre Factor $G$, Complejidad Cognitiva y Capacidad de Procesamiento ${ }^{1}$
}

\author{
Roberto Colom \\ Universidad Autónoma de Madrid \\ Carmen Flores-Mendoza ${ }^{2}$ \\ Universidade Federal de Minas Gerais
}

\begin{abstract}
RESUMEN - La psicologia experimental ha estudiado extensivamente las características de la memoria, mientras que la psicología correlacional ha actualizado las principales propiedades de la inteligencia. El acercamiento entre ambos enfoques se basa en el análisis de las relaciones entre un concepto nuclear de la memoria - la memoria de trabajo - y el principal ingrediente del concepto psicométrico de inteligencia - el factor $g$. Las evidencias disponibles sugieren que las diferencias individuales en $g$ podrían ser explicadas por los conceptos cognitivos de capacidad y de velocidad asociados a la memoria de trabajo. Esas evidencias sugieren nuevos modos de mejorar la inteligencia - y, por tanto, los correlatos asociados a ella - a través del incremento de la capacidad del sistema para procesar información de manera eficiente.
\end{abstract}

Palabras clave: inteligencia; diferencias individuales; memoria de trabajo; factor $g$.

\section{Intelligence and Working Memory: Relationship Between Factor $G$, Cognitive Complexity and Processing Capacity}

\begin{abstract}
Experimental psychology has studied memory characteristics extensively meanwhile correlational psychology has analyzed the main properties of intelligence. The approach between both viewpoints are based on memory nuclear concept analysis -working memory and, the main intelligence psychometrical concept $-g$ factor. The available data suggests that individual differences concern to the $g$ could be explained by cognitive concepts like span and speed linked to working memory. These data suggests new procedures to improve intelligence - and therefore the social aspects associated to it - by increasing system capacity of efficient information processing.
\end{abstract}

Key words: intelligence; individual differences; working memory; $g$ factor.

\section{Inteligência e Memória de Trabalho: A Relação Entre Fator $G$, Complexidade Cognitiva e Capacidade de Processamento}

\begin{abstract}
RESUMO - A psicologia experimental tem estudado extensivamente as características da memória, enquanto que a psicologia correlacional tem analisado as principais propriedades da inteligência. A aproximação entre ambos enfoques baseia-se na análise das relações entre um conceito nuclear da memória - a memória de trabalho - e o principal ingrediente do conceito psicométrico de inteligência - o fator $g$. As evidências disponíveis sugerem que as diferenças individuais em $g$ poderiam ser explicadas pelos conceitos cognitivos de capacidade e de velocidade associados à memória de trabalho. Essas evidências sugerem novos modos de melhorar a inteligência - e, portanto, os correlatos associados a ela - através do incremento da capacidade do sistema para processar informação de maneira eficiente.
\end{abstract}

Palavras-chave: inteligência; diferenças individuais; memória de trabalho; fator $g$.

\section{La Memoria Humana}

La memoria constituye un atributo humano basado en el hecho de que las personas son capaces de almacenar infor-

1 Manuscrito elaborado por ocasión de la estancia realizada por Carmen Flores-Mendoza en el Departamento de Psicología Biológica y de la Salud de la Universidad Autónoma de Madrid con la supervisión de Roberto Colom. Agradecimiento especial al profesor Dr. Adail Victorino Castilho, de la Universidad de São Paulo, por los comentarios a un borrador de este artículo, gracias a los cuales se han podido corregir algunas imprecisiones y mejorar buena parte de los argumentos y presentación de evidencias.

2 Dirección: Departamento de Psicología, FAFICH-UFMG, Sala 4042, Av. Antonio Carlos, 6627 CEP: 31270-901

E-mail:carmenflor@uol.com.br mación para utilizarla posteriormente. Si se nos pregunta el significado de la palabra "radiación", seremos capaces de explicar que se trata de ondas emitidas por determinados materiales. Para poder responder de este modo, es necesario que hayamos almacenado en el pasado el significado de esa palabra. Pero, ¿cómo se aprende y se almacena ese significado? ¿dedicamos las personas 10 minutos al día para consultar un diccionario enciclopédico y tratar de almacenar el significado de más y más palabras? La respuesta es negativa: el significado de las palabras suele aprenderse a través de mecanismos de inferencia que permiten deducir su significado a partir del contexto. Por tanto, aprender el significado de las palabras es un proceso activo y en cierto modo no explícito. ¿Cómo podemos recuperar el significado de esas 
palabras? ¿Cuáles son los procesos mentales que nos permiten recuperar el significado de las palabras o cualquier otro tipo de información? Para intentar comprender en qué consiste la memoria, los psicólogos han ideado paradigmas experimentales que han permitido poner a prueba, en los laboratorios, ese atributo humano. Estos paradigmas han servido para contrastar una serie de teorías sobre la memoria humana.

En Psicología existen, al menos, tres grandes teorías de la memoria (Smith, 1996): sobre las etapas de memoria, sobre los sistemas de memoria y basadas en los recursos de la memoria. Estas teorías poseen sus ventajas e inconvenientes, en el sentido de que son congruentes con algunas evidencias experimentales, pero incongruentes con otras. Veamos brevemente una caracterización de estas teorías.

Las teorías sobre las etapas de la memoria distinguen la fase de codificación, de almacenamiento y de recuperación. La codificación se centra en la percepción de la información, el almacenamiento consiste en el mantenimiento de esa información y la recuperación se basa en el recuerdo de la información previamente almacenada.

Las teorías sobre los sistemas de memoria quizá son las más conocidas. En ellas se suele distinguir entre la memoria sensorial (MS), la memoria a corto plazo (MCP) y la memoria a largo plazo (MLP). Aquí se supone que el sujeto recibe la información del entorno a través de los órganos de los sentidos, dando lugar a impresiones sensoriales que se almacenan en la MS, pero cuya duración no suele exceder el medio segundo. Gran parte de la información incluida en esas impresiones sensoriales, se desvanece rápidamente. La información superviviente se traslada desde la MS a la MCP, donde el sujeto ya pueda manipularla conscientemente. Al igual que la MS, la MCP también presenta limitaciones temporales, y, por tanto, la información tiende a perderse, bien por desvanecimiento, bien porque la llegada de nueva información desplaza a la existente. Parte de la información procesada en la MCP se traslada a la MLP, también denominada memoria permanente. La información que se almacena en la MLP se distingue en varios tipos: episódica, semántica, procedimental, etc. Esta información almacenada puede ser recuperada posteriormente.

Finalmente, las teorías basadas en los recursoscognitivos se centran en explicar los procesos cognitivos de memorización, a partir de parámetros tales como la velocidad con la que se puede procesar mentalmente la información, la capacidad de la MCP o la habilidad para inhibir el procesamiento mental de la información irrelevante. Desde estas teorías, las limitaciones temporales de la MCP conllevan que el sujeto puede procesar una determinada cantidad de información por unidad de tiempo. Cuando un sujeto puede procesar la información de la MCP a mayor velocidad que otro, entonces posee una ventaja, en el sentido de que podrá procesar más información por unidad de tiempo.

No es éste el lugar de hacer una presentación exhaustiva sobre la memoria humana. Existen excelentes tratados al respecto (Anderson, 1995; Baddeley, 1997; Estes, 1987). En ellos se pueden encontrar los distintos modelos de memoria humana que se han presentado hasta la fecha. No obstante, con el ánimo de centrar la discusión, se optará aquí por un modelo de memoria propuesto por E.B. Hunt en 1971. Este modelo ha sido empleado por J.B. Carroll (1993) entre otros, para interpretar en términos cognitivos las diferencias de aptitud. Ello justifica en parte nuestra elección.

El modelo de memoria de Hunt (1971) distingue entre componentes básicos de la memoria y procesos de control.

Los componentes básicos son: el retén o buffer sensorial, equivalente a la memoria sensorial, la Memoria a Corto Plazo (MCP), la Memoria a Medio Plazo (MMP) y la Memoria a Largo Plazo (MLP). En una conversación, la MCP recuerda palabra por palabra, la MMP recuerda un esbozo general del tema de la conversación y la MLP conserva lo que se conoce sobre cada palabra más allá de la conversación concreta que se está manteniendo. Los procesos de control son: los procesos atencionales que permiten trasladar la información desde el retén sensorial a la MCP, los procesos de repaso que permiten evitar la pérdida de información en la MCP, los procesos de formación de bloques de información que ayudan a mantener más cantidad de información en la MCP agrupando las piezas de información en unidades compactas, los procesos de codificación que permiten trasladar la información desde la MCP a la MLP y los procesos de búsqueda en la MLP que permiten recuperar información almacenada en la MLP para ser manipulada en la MCP.

Las investigaciones han permitido demostrar que existen notables diferencias individuales en el uso de estos procesos de control, y, por tanto, en los procesos de memorización (Carroll, 1993). Veamos un ejemplo:

Condición 1 (identificación física):

(1) Se presentan en la pantalla de un ordenador dos letras (A a).

(2) El sujeto debe decidir lo antes posible, y sin equivocarse, si las dos letras son idénticas (en este caso, el sujeto responderá que NO son idénticas).

(3) Se repite esta presentación $n$ veces y se calcula el tiempo medio que consume cada sujeto al tomar la decisión de si las dos letras son idénticas o no lo son.

Condición 2 (identificación semántica):

(1) Se presentan en la pantalla de un ordenador dos letras (A a).

(2) El sujeto debe decidir si las dos letras poseen el mismo significado (en este caso, el sujeto responderá que SI poseen el mismo significado).

(3) Se repite esta presentación $n$ veces y se calcula el tiempo medio que consume cada sujeto al tomar la decisión de si las dos letras significan lo mismo.

Es fácil suponer que, en promedio, los sujetos tardarán más tiempo en responder a la Condición 2 que a la Condición 1. ¿Por qué? El modelo de memoria de Hunt (1971) ayuda a encontrar una respuesta. En la Condición 1, el sujeto sólo necesita introducir las dos letras en su MCP, realizar una comparación de la apariencia física de las dos letras y 
responder. En cambio, en la Condición 2, el sujeto necesita introducir las dos letras en su MCP, realizar una comparación de la apariencia física de las dos letras, recurrir a su MLP para recuperar el significado de las dos letras y responder. Por tanto, hay un paso más en la Condición 2 que en la Condición 1: recuperar el significado de las dos letras desde la MLP. Puesto que los procesos mentales exigen un tiempo, y dado que la Condición 2 requiere un proceso mental más que la Condición 1, entonces resulta lógico pensar que el tiempo empleado para responder será mayor en la Condición 2 que en la Condición 1. Empleando el método de sustracción, habitual en los estudios sobre cronometría mental (Smith \& Jonides, 1997) se puede restar el tiempo empleado en la Condición 1 del tiempo empleado en la Condición 2 para calcular el tiempo necesario para recuperar el significado de las dos letras desde la memoria permanente.

Hunt, Lunneborg y Lewis (1975) han estudiado a sujetos con distintas puntuaciones en tests estandarizados de aptitud verbal, comprobando que:

(1) Los sujetos con altas y bajas puntuaciones en esos tests emplean un tiempo equivalente en la Condición 1. Es decir, poseer una alta aptitud verbal no conlleva realizar una comparación física más rápida.

(2) Los sujetos con mayores puntuaciones en esos tests emplean menos tiempo en la Condición 2 que los sujetos con menores puntuaciones. Por tanto, las diferencias individuales en la aptitud verbal correlacionan con la velocidad con la que se pueden realizar comparaciones semánticas.

(3) Los sujetos con mayores puntuaciones emplean menos tiempo en recuperar el significado de las dos letras desde su MLP. Las diferencias individuales en aptitud verbal correlacionan con las diferencias individuales en la velocidad de recuperación de significados desde la MLP. Concretamente, los sujetos de menor aptitud verbal tardan 3 veces más que los de mayor aptitud verbal en recuperar esos significados (86 milisegundos frente a 33 milisegundos).

En suma, cuando se considera el rendimiento de los sujetos en las tareas experimentales que permiten estudiar algunos de los procesos asociados a la memoria humana, se observa que existen notables diferencias individuales. Pero, ¿son todas esas diferencias individuales igualmente relevantes? ¿cuáles son los procesos de memoria en los que se aprecian diferencias individuales más significativas en términos psicológicos? Actualmente tiende a pensarse que esas diferencias resultan más informativas cuando se relacionan con los mecanismos de almacenamiento y procesamiento de la MCP, denominada en los últimos años memoria de trabajo (working memory).

\section{Memoria Humana y Inteligencia}

¿Dónde encaja la memoria humana dentro de los modelos o teorías de la inteligencia? Responder a esta pregunta exige considerar los modelos estructurales de la inteligencia. Según la Asociación Americana de Psicología (Neisser y cols., 1996) estos son los modelos más robustos actualmente disponibles sobre la inteligencia.

La inteligencia se suele definir como una capacidad mental muy general que implica la aptitud para razonar, planificar, resolver problemas, pensar de modo abstracto, comprender ideas complejas, aprender con rapidez y aprender de la experiencia. Además, no se puede considerar un mero conocimiento enciclopédico, una habilidad académica particular o una pericia para resolver tests. La inteligencia refleja una capacidad más amplia y profunda para comprender el ambiente-darse cuenta, dar sentido a las cosas o imaginar qué se debe hacer. Desde esta perspectiva, la inteligencia se suele concebir como una capacidad integradora de la mente (Colom \& Andrés Pueyo, 1999; Juan-Espinosa, 1997).

Aunque en el transcurso del presente siglo se han propuesto varios modelos sobre la estructura de la inteligencia, actualmente se toma como punto de referencia obligada el de J.B. Carroll (1993): la teoría de tres estratos (Three Stratum Theory). Esta teoría es resultado de analizar 461 estudios de 19 países distintos, con más de 130.000 sujetos. ${ }^{3}$ En ella se distingue entre aptitudes concretas, amplias y generales. El estrato en el que se sitúa una determinada aptitud es signo de su grado de generalidad. Estas aptitudes constituyen un reflejo de las diferencias individuales que se pueden observar cuando las personas realizan pruebas psicológicas intelectualmente exigentes (similares a las incluidas en los tests estandarizados de inteligencia).

En el tercer estrato se sitúa $g$, o inteligencia general, en el segundo estrato se sitúan una serie de aptitudes amplias y en el primer estrato se sitúan las aptitudes específicas. De entre las aptitudes amplias hay una que se corresponde con el dominio de la memoria (2Y) dentro de la que caen una serie de aptitudes específicas: amplitud de memoria (MS), memoria asociativa (MA), recuerdo libre (M6), memoria semántica (MM) y memoria visual (MV).

Según esta teoría, la memoria constituye una aptitud amplia, cuya importancia como marcador de la inteligencia general $(g)$ solamente es superada por la inteligencia fluida (2F) y la inteligencia cristalizada (2C). El análisis de Carroll (1993) lleva a dos conclusiones generales sobre la memoria:

(1) Los individuos difieren en una aptitud general de memoria que afecta al rendimiento en una variedad de tareas y conductas que suponen memorizar.

(2) Hay evidencia de aptitudes más específicas dentro de la aptitud de memoria (las aptitudes específicas señaladas). La amplitud de memoria (MS) supone la cantidad de material (verbal, numérico, o figurativo) que puede recordar un individuo de manera inmediata, en el orden en el que ha sido presentada. La memoria asociativa (MA) es la capacidad para formar asociaciones arbitrarias sobre un determinado material estimular; la persona debe recordar o reconocer qué estímulos se encuentran asociados. En cuanto al recuerdo libre (M6) se sabe que algunas personas, tras una

3 Un análisis del extensivo estudio de Carroll (1993) puede verse en Andrés Pueyo (1997), Colom (1998) o Juan-Espinosa (1997). 
fase de estudio, son capaces de recordar más que otras personas en una fase de examen, cuando la cantidad de material que se ha de recordar supera la amplitud promedio de memoria de una persona estándar (vg. el promedio). En cuanto a la memoria semántica (MM) determinadas personas, después de una fase de estudio, son capaces de reconocer o recordar más material que otras personas, siempre que el material guarde una relación significativa. La memoria visual (MV) es la aptitud para formar, durante una fase de estudio, una representación mental del material que se presenta, cuando el material visual no se puede codificar en alguna otra modalidad. La persona debe usar dicha representación para responder en una fase de prueba, recordando o reconociendo el material.

En suma, la memoria forma parte de los modelos estructurales de la inteligencia y constituye, tanto una aptitud amplia, como un conglomerado de aptitudes específicas. El análisis factorial ha permitido identificar estas aptitudes vinculadas a la memoria, gracias a que se han podido detectar diferencias individuales en las tareas que se han empleado para medir distintos aspectos relacionados con las actividades vinculadas a los procesos de memorización. La memoria es, por tanto, un componente más de la inteligencia humana. Hasta qué punto determinados aspectos de la memoria son o no son centrales para explicar las diferencias de inteligencia, es algo que se tratará de dilucidar en este artículo.

\section{La memoria de trabajo (working memory)}

El constructo "memoria de trabajo" está inspirado en los modelos estructurales de memoria en los que se distinguía, como ya se ha comentado, la MS, la MCP y la MLP. Originalmente, la MCP se concebía como un almacén en el que se retenía la información durante unos segundos. La investigación de la MCP permitió descubrir una serie de estrategias usadas por los sujetos para procesar la información: repaso, repetición, organización y algunos más (FloresMendoza \& Colom, en prensa).

El estudio experimental de la MCP dio lugar a que Baddeley (1986) profundizase en el constructo de "memoria de trabajo", distinguiendo el almacenamiento transitorio de la información y su procesamiento bajo la supervisión de un ejecutivo central. La mayor parte de los estudios se han centrado en el análisis de las características de dos sistemas de almacenamiento transitorio de la información, el bucle articulatorio y la agenda visoespacial (Baddeley, 1997; Baddeley, Lewis \& Vallar, 1984; Baddeley \& Wilson, 1985).

En los últimos años se ha tratado de organizar las tareas que se han empleado para estudiar la memoria de trabajo. Oberauer y cols. (2000) han distinguido, en este sentido, las funciones de los recursos de la memoria de trabajo y el dominio de contenido de la tarea. En cuanto a las funciones de las memoria de trabajo se distinguen: a) Las de almacenamiento y procesamiento (Salthouse, 1990). Estas funciones exigen, por un lado, mantener activos en un estado accesible los contenidos mentales, y, por otro lado, transformar los contenidos a través de las operaciones mentales, b) Las de supervisión suponen tutelar y controlar las operaciones y acciones mentales (Dempster, 1992), y c) Las de coordinación pueden suponer tres cosas: coordinar la información de diferentes fuentes (Morrin, Law \& Pellegrino, 1994), coordinar operaciones mentales sucesivas en una secuencia (Hagendorf \& Sá, 1995) y, coordinar los elementos en estructuras (Oberauer, 1993). En cuanto al dominio de contenido de la tarea, esta suele ser verbal, numérica y figurativa. Según Oberauer y cols. (2000) "la diferenciación de la memoria de trabajo según la dimensión de contenido se ha estudiado en alguna medida, pero sigue siendo un problema no resuelto" (p. 1020).

Los resultados del estudio de Oberauer y cols. (2000) indican que:

(1) Las funciones de procesamiento y almacenamiento de información son inseparables de las funciones de coordinación.

(2) Las funciones de supervisión están relacionadas con la velocidad de procesamiento.

(3) Las tareas figurativas son claramente distintas de las verbales y numéricas.

En ocasiones se ha sugerido que la memoria de trabajo se puede concebir como un mecanismo de propósito general que limita los recursos de procesamiento de los que dispone el sujeto. Por tanto, la memoria de trabajo comprometerá todas las tareas que el sujeto deba realizar (Colom \& Flores-Mendoza, enviado; Colom, Palacios, Kyllonen \& Juan-Espinosa, enviado; Kyllonen \& Christal, 1990; Smith, 1996). Sin embargo, otros autores discrepan. En este sentido, Carpenter y Just (1988) comentan que

la capacidad de la memoria de trabajo no se puede concebir como una propiedad general de una estructura fija. Además, la aproximación operacional sugiere que no existe una medida absoluta de la capacidad de la memoria de trabajo; solo se puede medir con respecto a una serie de operaciones mentales dentro de un determinado dominio. Desde este punto de vista, no sería sorprendente que la capacidad de la memoria de trabajo medida a través de una tarea, no fuese predictiva del rendimiento en otra tarea distinta (p. 22).

Años después, Just y Carpenter (1992) escriben en la misma línea:

en suma, no podemos concluir que la capacidad de la memoria de trabajo usada para la comprensión lingüística sea la única capacidad cognitiva. Por el contrario, es probable que exista un amplio conjunto de recursos de procesamiento, de los que solamente algunos se emplean al resolver un determinado tipo de tareas (p. 144, cursiva nuestra).

En su revisión sobre el estado de la cuestión, Logie (1996) concluye dos cosas:

(a) La hipótesis de un mecanismo de propósito general en la memoria de trabajo es muy improbable.

(b) La estructuración del conocimiento en determinados dominios, diferentes para cada persona, facilitaría las transformaciones y la recuperación de ésta información de la memoria permanente. 
En consecuencia, la memoria de trabajo constituye un sistema de memoria en el que los sujetos almacenan transitoriamente la información y la someten a procesamiento. Esa información puede provenir tanto de una determinada tarea como de la MLP. El resultado de ese procesamiento dará como consecuencia la respuesta del sujeto. No obstante, sigue siendo un problema no resuelto si la memoria de trabajo se puede concebir como un mecanismo de propósito general, o, si, por el contrario, está compuesta por distintos subsistemas.

\section{El factor $g$}

El rendimiento de las personas ante diversos problemas tiende a relacionarse positivamente. Esta correlación positiva constituye un fenómeno natural que ha dado lugar al concepto de factor $g$. Este factor general, $g$, se puede extraer a partir de una matriz de correlaciones compuesta por una serie de tests de aptitud (Carroll, 1993; Jensen, 1998). En la Tabla 1 se presenta una matriz de correlaciones que puede ser explicada por un sólo factor $(g)$. Cuando se calcula un análisis factorial sobre esta matriz de correlaciones, se observa que únicamente es necesario una dimensión o factor para explicar el patrón de las correlaciones observadas entre los tests. Nótese, además, que los valores en $g$ de los distintos tests es diferente: el test 1 presenta un peso de 0.90 , mientras que el test 6 presenta un peso de 0.40 . Ello se debe a que el test 1 es el que más correlaciona con todos los demás tests, mientras que el test 6 es el que menos correlaciona con todos los demás tests. Ese hecho lleva a sostener que el test 1 está midiendo mejor la inteligencia que el test 6: una buena medida de la inteligencia presenta mayores pesos en $g$. Cuanto menor sea ese peso, peor se mide la inteligencia y mejor se miden habilidades específicas vinculadas a la resolución de ese test en particular.

Esto ocurre con una matriz ideal, pero ¿se encuentra también con las matrices de correlaciones reales? En otras palabras, ¿es $g$ empíricamente importante o simplemente es resultado de aplicar la técnica estadística del análisis factorial? ¿es $g$ un fenómeno natural o constituye un artefacto estadístico?

En la Tabla 2 se presenta el análisis factorial jerárquico (transformación Schmid-Leiman) aplicado especialmente para este artículo, a la matriz de correlaciones obtenida a partir de los 14 subtests incluidos en la escala Wechsler para

Tabla 1. Una ideal matriz de correlaciones que puede ser reducida a una sola dimensión común a todas las medidas o tests. Esta dimensión común se suele denominar $g$

\begin{tabular}{lcccccc}
\hline Tests & $\mathbf{1}$ & $\mathbf{2}$ & $\mathbf{3}$ & $\mathbf{4}$ & $\mathbf{5}$ & $\mathbf{6}$ \\
\hline 1 & & +0.72 & +0.63 & +0.54 & +0.45 & +0.36 \\
2 & & & +0.56 & +0.48 & +0.40 & +0.32 \\
3 & & & & +0.42 & +0.35 & +0.28 \\
4 & & & & & +0.30 & +0.24 \\
5 & & & & & & +0.20 \\
6 & & & & & & \\
\hline Peso en $g$ & +0.90 & +0.80 & +0.70 & +0.60 & +0.50 & +0.40 \\
\hline
\end{tabular}

Tabla 2. Análisis factorial jerárquico (transformación Schmid-Leiman) a partir de la matriz de correlaciones compuesta por los subtests del WAIS III

\begin{tabular}{lrrrr}
\hline Subtest del WAI S III & $\boldsymbol{g}$ & $\boldsymbol{F 1}$ & $\boldsymbol{F 2}$ & $\boldsymbol{F 3}$ \\
\hline (1) Vocabulario & 0.78 & 0.02 & 0.39 & 0.08 \\
(2) Semejanzas & 0.76 & 0.04 & 0.36 & 0.08 \\
(3) Aritmética & 0.76 & 0.08 & 0.13 & -0.11 \\
(4) Dígitos & 0.76 & 0.01 & 0.00 & -0.32 \\
(5) Información & 0.75 & 0.02 & 0.35 & 0.05 \\
(6) Comprensión & 0.71 & -0.05 & 0.43 & 0.07 \\
(7) Letras/Números & 0.85 & 0.07 & -0.02 & -0.31 \\
(8) Figuras incompletas & 0.74 & 0.27 & 0.11 & 0.08 \\
(9) Clave Números & 0.77 & 0.33 & -0.06 & -0.04 \\
(10) Cubos & 0.76 & 0.37 & 0.00 & 0.07 \\
(11) Matrices & 0.82 & 0.32 & 0.05 & 0.05 \\
(12) Historietas & 0.77 & 0.29 & 0.07 & 0.06 \\
(13) Símbolos & 0.75 & 0.35 & -0.06 & 0.00 \\
(14) Rompecabezas & 0.71 & 0.35 & 0.00 & 0.08 \\
\hline \% Varianza & 58.43 & 5.5 & 4.5 & 1.86 \\
\hline
\end{tabular}

adultos (WAIS III), recientemente adaptada para población española (TEA, 1998). Las características de la estandarización española del WAIS III son: $\mathrm{N}=1369$; Rango de edad: 15 a 94 años; Sexo: 703 mujeres y 666 hombres; Región: 348 Norte, 299 Centro, 359 Este, y 363 Sur; Zona: 659 Urbana, 234 Rural, y 476 Intermedia; Nivel de estudios: 301 sin estudios, 432 primer grado, 525 segundo grado, y 111 tercer grado. Por tanto, se trata de una muestra representativa de la población española.

¿Qué nos indica la matriz factorial de la Tabla 2? Una de las principales evidencias es que el factor $g$, representante estadístico de la inteligencia general, explica la mayor parte de la información incluida en la matriz de correlaciones. Obsérvese el porcentaje de varianza, que es de más del 58\% para $g$, y oscila entre 5.5 y 1.86 para los factores más específicos, F1, F2, y F3. Entre los 3 factores específicos se explica algo menos del $12 \%$ de la varianza, de modo que $g$ explica 5 veces más varianza que los tres factores específicos juntos. Por tanto, la importancia o no de $g$ es algo que debe dilucidarse empíricamente. Y cuando esto se hace, el resultado es claro y contundente: $g$ es empíricamente importante.

Existen distintos métodos para obtener $g$ a partir de una matriz de correlaciones. Es decir, se pueden emplear distintos métodos de análisis factorial, pero todos ellos permiten obtener un $g$ prácticamente idéntico. En otras palabras, $g$ no cambia cuando se emplean distintos métodos de análisis factorial, siempre y cuando se cumplan unos criterios mínimos: muestras representativas y amplias, así como tests variados (y en un número suficientemente amplio) que reclamen distintas actividades cognitivas del sujeto (Colom y cols., 2000; Jensen, 1998; Jensen \& Weng, 1994).

Aunque son varios los tests que, de un modo consistente, presentan una mayor carga de $g$, es decir, son mejores medidas de $g$, resulta conceptualmente incorrecto considerar que las características de esos tests (p.e. el establecimiento de relaciones o el razonamiento abstracto) constituyen la esencia de $g$. La carga en $g$ de un test y su nivel de dificultad, son aspectos conceptualmente separados. También es inade- 
cuado considerar a $g$ como un proceso cognitivo, como un principio operativo de la mente o como una característica del diseño de los circuitos neuronales del cerebro. A nivel psicométrico, $g$ debe concebirse como una fuente común de diferencias individuales en todos los tests mentales. En este sentido, $g$ se podría equiparar a la potencia, eficacia o velocidad de la CPU de un ordenador. El conocimiento y las habilidades que exigen los tests constituyen un modo de medir $g$, pero no son $g$ (Jensen, 1998).

El fenómeno de las correlaciones positivas observadas empíricamente cuando se consideran tests cognitivos, ha dado lugar a dos principios básicos asociados al fenómeno psicológico de la inteligencia:

(1) $G$ no se relaciona con el contenido específico de los problemas de los tests o con sus características superficiales. Este constituye el principio de la indiferencia del indicador.

(2) $G$ se relaciona con la complejidad de la actividad cognitiva exigida por los problemas, es decir, captar relaciones entre elementos, conceptos abstractos, razonar, analizar, hallar características comunes entre elementos superficialmente distintos e inferir conclusiones a partir de los elementos de información.

Una de las principales razones por las que en ocasiones se considera que los tests de inteligencia no pueden ser importantes para predecir el rendimiento en la vida diaria (p.e. en la escuela o las distintas ocupaciones) es que sus ítems a menudo parecen alejados de esa vida. Sin embargo, ello no es más que una apariencia. El contenido de los tests es lo menos importante. La clave está en su complejidad. Un ejemplo lo tenemos en el subtest de dígitos del WAIS: con exactamente el mismo tipo de contenido se puede convertir el subtest en una tarea más o menos compleja (cognitivamente hablando). La versión más compleja (recuerdo inverso) tiene más que ver con la inteligencia general (factor $g$ ) que la versión más simple (recuerdo directo). En el caso de la baremación española del WAIS III, el peso en $g$ de dígitos directo es .65 , mientras que el peso de dígitos inverso es de .736 .

Otro ejemplo lo podemos observar en el nivel de vocabulario. Aunque pudiera parecer que la riqueza de vocabulario deriva de una simple exposición pasiva a las palabras y a su significado, realmente el elemento clave está en un proceso de inferencia de significados (adquirir un vocabulario supone un proceso de distinción y generalización de conceptos). Por tanto, el contraste, la abstracción, la inferencia, la búsqueda de similitudes y diferencias, constituyen algunas de las piezas claves de la inteligencia, tal y como se manifiesta en el razonamiento, la solución de problemas y la adquisición de nuevos conceptos.

Los problemas más cargados de $g$ son aquellos que exigen razonamiento deductivo o inductivo, visualización espacial, razonamiento cuantitativo, y conocimiento y razonamiento verbal (significado de las palabras, distinciones entre palabras relacionadas, sinónimos-antónimos, analogías verbales y comprensión lectora). Los mejores problemas de $g$ imponen exigencias mínimas de conocimiento especializado. Estas características de los problemas a través de los que se mide $g$ (los llamados "vehículos de $g$ ") son compartidas con muchas actividades cotidianas, de ahí su importante validez predictiva (Colom \& Andrés-Pueyo, 1999).

Hay que destacar que el factor $g$ es compatible con la existencia de un enorme número de aptitudes intelectuales. De este modo, la investigación de la inteligencia sugiere que ésta no es "única". Al contrario, la inteligencia, como concepto científico, estaría compuesta por más de 60 aptitudes (Carroll, 1993). El factor $g$ sería resultado de los elementos comunes a esa serie de aptitudes. Según Carroll (1997) $g$ explicaría más de la mitad de la varianza contenida en una matriz de correlaciones, hecho que suele tomarse como síntoma de su importancia, tanto conceptual como práctica.

\section{Inteligencia y Memoria de Trabajo}

Los psicólogos cognitivos han explorado la relevancia del constructo "memoria de trabajo" para la explicación de las diferencias individuales en tareas cognitivamente exigentes que varían en su nivel de complejidad. Así, por ejemplo, el equipo del Carnegie Mellon, dirigido por Carpenter y Just, ha estudiado la relevancia de las restricciones de capacidad de la memoria de trabajo para explicar las diferencias individuales al resolver tareas figurativas complejas, como el Test de Matrices Progresivas de Raven, o tareas de Comprensión Lingüística en las que se manipula el nivel de complejidad del material verbal a comprender.

Carpenter, Just y Shell (1990) analizaron las diferencias individuales en el Test de Matrices de Raven, según la tesis de las restricciones de capacidad de la memoria de trabajo. Las matrices de Raven exigen un procesamiento cognitivo complejo de la información, es decir, demandan una gran cantidad de recursos cognitivos. Estos autores escribieron dos programas de ordenador, FAIRAVEN y BETTERAVEN, que simulaban la conducta de los sujetos de medio y alto rendimiento, respectivamente. BETTERAVEN difería de FAIRAVEN en que disponía de un supervisor de objetivos, equivalente al ejecutivo central de la memoria de trabajo. Este supervisor capacita a los sujetos de alto rendimiento para gestionar los análisis simultáneos que necesariamente deben realizarse para resolver los ítems más complejos del Raven, mientras que su carencia produce un colapso informativo cuando los sujetos de rendimiento medio tratan de resolver las matrices más complejas.

Just y Carpenter (1992) han propuesto una teoría de la comprensión lingüística restringida por la capacidad a partir de un amplio número de resultados experimentales. Según esos resultados, los sujetos con una mejor comprensión son capaces de mantener en un estado activo durante más tiempo las piezas de la frase a comprender, de modo que su procesamiento resulta más preciso (es decir, una mejor comprensión exige más tiempo de procesamiento). En contraste, las personas con una peor comprensión muestran claras dificultades para conservar activo el significado de las distintas piezas de la frase, es decir, manifiestan déficits de capacidad (además, tardan menos tiempo en responder). A medida que aumenta la complejidad de las frases que se de- 
ben comprender, se incrementan las diferencias individuales, de modo que los sujetos con mayor capacidad logran mantener un óptimo nivel de eficacia, mientras que los sujetos de menor capacidad reducen su rendimiento significativamente. Es importante destacar que los sujetos con mejor comprensión no difieren de los de peor comprensión por que los primeros emplean algunos procesos cognitivos que los segundos no emplean: las diferencias individuales radican en la capacidad para almacenar y procesar transitoriamente la información necesaria para poder comprender los mensajes lingüísticos (véase un comentario crítico en Waters \& Caplan, 1996, y la réplica en Just, Carpenter \& Keller, 1996). Se trata, por tanto, de una diferencia básica de la capacidad de la memoria de trabajo, es decir, de la disponibilidad para soportar los colapsos producidos por sobrecarga de información o de necesidades de procesamiento.

Estos resultados sugieren que las diferencias individuales en este tipo de tareas se pueden explicar, al menos en parte, por las restricciones de capacidad de la memoria de trabajo. Así, los sujetos con una alta capacidad pueden supervisar eficientemente el procesamiento de la información, mientras que los sujetos de baja capacidad no disponen de los recursos necesarios para realizar esta supervisión. Por tanto, la memoria de trabajo constituiría un módulo de recursos para procesar información que limita la capacidad del sujeto para resolver tareas cognitivamente exigentes que varían en su nivel de complejidad: cuanto más compleja es la tarea, mayor es la importancia de estas restricciones de capacidad.

La capacidad de la memoria de trabajo se ha relacionado con el concepto central de la inteligencia estudiada a través de los tests estandarizados: el factor $g$. Junto con la memoria de trabajo se ha considerado la velocidad de procesamiento. Según Jensen (1993):

si existe un componente básico del sistema de procesamiento humano de información que influya sobre la varianza de $\mathrm{g}$, la mayor parte de los teóricos cognitivos estarán de acuerdo en que es la memoria de trabajo, un constructo hipotético concebido como una unidad central de procesamiento de información (pág. 122, cursiva nuestra).

Refiriéndose también a la velocidad de procesamiento, el mismo autor escribe que:

la mayor parte de los teóricos cognitivos creen que al menos son necesarias dos variables fundamentalmente distintas para explicar g: la velocidad del procesamiento de la información, y la capacidad de la memoria a corto plazo (incluyendo la memoria de trabajo) ... la capacidad parece ser un concepto necesario para comprender $\mathrm{g}$, dado que las personas difieren en su aptitud al resolver tareas que imponen más y menos demandas a la memoria de trabajo (pág. 123, cursiva nuestra).

Basándose en esta perspectiva, tanto Jensen como otros autores, han empleado las denominadas tareas cognitivas elementales (TCEs) ${ }^{4}$ para intentar comprobar si las limita-

4 Conocidas en inglés como Elementar Cognitive Tasks ó simplemente ECTs. ciones de la memoria de trabajo y de la velocidad de procesamiento se asocian con $g$. Estas TCEs intentan evaluar una serie de procesos cognitivos simples, independientemente del conocimiento específico o del contenido de la información. Las diversas TCEs intentan explorar distintos procesos, tales como la captación del estímulo, la discriminación, la elección, la búsqueda visual, el rastreo de la memoria a corto plazo y la recuperación de información desde la memoria a largo plazo. La mayor parte de la TCEs son tan simples que cualquier persona puede realizarlas sin presentar errores. Las diferencias individuales se consignan en virtud del tiempo de reacción. Las TCEs más interesantes son aquellas que exigen menos de 1 segundo para responder y en las que el nivel de error está próximo a 0.

En las TCEs, las variables que se registran son: a) el tiempo de reacción (TR), que es el intervalo temporal entre la aparición del estímulo y el momento en que el sujeto deja de presionar un botón de salida; b) el tiempo de movimiento (TM), que es el intervalo temporal entre dejar de presionar el botón de salida y la presión del botón de respuesta, y c) la puntuación de error, esto es, si el sujeto responde correcta o incorrectamente. A partir de estas tres medidas básicas se obtienen cuatro variables de diferencias individuales: 1) TR mediano; 2) la desviación típica del TR del sujeto en una serie de ensayos (DTTR); 3) TM mediano; 4) la desviación típica del TM del sujeto en una serie de ensayos (DTTM).

Veamos un ejemplo de TCEs con fines ilustrativos. En la siguiente secuencia se combina el paradigma experimental clásico de rastreo de memoria (Memory scan) y una tarea de sinónimos-antónimos:

1) Señal preparatoria

2) Pantalla en blanco

3) 72513 (serie de dígitos)

4) CALIENTE-FRIO (antónimos?)

5) El sujeto presiona el botón $\quad \mathrm{ATR}_{1} \mathrm{y} \mathrm{TM}_{1}$

6) Pantalla en blanco $1 \mathrm{seg}$.

7) 5 (dígito de test)

8) El sujeto presiona el botón SI $\mathrm{TR}_{1}$ y $\mathrm{TM}_{2}$

El sujeto debe conservar la serie de dígitos en su memoria (3), mientras procesa el significado de las palabras (4 y $5)$, de modo que cuando aparece el dígito de test (7) debe rastrear su memoria para poder responder (8). El aumento de la "amenaza" sobre la memoria de trabajo, incrementa tanto el $\mathrm{TR}_{1}$ como el $\mathrm{TR}_{2}$.

Las correlaciones de las TCEs con las medidas de inteligencia oscilan entre - .10 y - .50 ( -.35 en promedio) dependiendo de la complejidad o del número de procesos reclamados por la TCEs (Jensen, 1998). Algunos procesos correlacionan más con la inteligencia. Las TCEs que ponen a prueba la capacidad de la memoria de trabajo presentan mayores correlaciones con las puntuaciones de Cociente Intelectual (CI). Una puntuación compuesta de TR y DTTR en distintas TCEs, permite muestrear un mayor número de procesos cognitivos, y produce correlaciones entre -.5 y -.7 con las medidas de CI.

La Figura 1 (adaptada de Jensen, 1998), presenta un resumen de los resultados obtenidos con las TCEs: se observa 


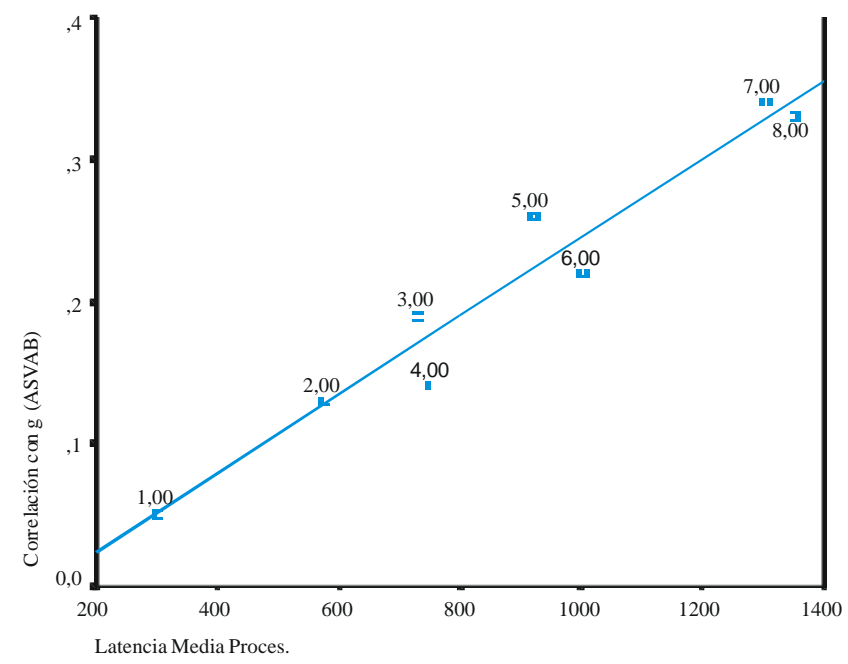

Figura 1. Resumen de resultados de la correlación entre g y TCEs que varían en su nivel de complejidad. Se observa que a mayor complejidad de la TCEs, mayor es la correlación con $g$.

que a mayor complejidad de la TCE, mayor es la correlación con las estimaciones psicométricas de la inteligencia general $(g)$.

Los resultados observados en la Figura 1 son congruentes con los hallados en un estudio realizado por FloresMendoza (1999) en Brasil: tanto el TR como el número de errores cometidos por sujetos con retraso mental eran sustancialmente mayores en tareas de TR de elección que en tareas de TR simple. Además, y este es el punto importante, las correlaciones entre el TR obtenido en las tareas de TR de elección y las puntuaciones alcanzadas en el Test de Matrices de Raven variaban entre $-0,46$ y $-0,61$ (-0,5 en promedio). Sin embargo, las correlaciones entre el TR simple y el Raven no resultaron significativas. Por tanto, cuanto mayor es la complejidad del procesamiento cognitivo exigido, mayores son las relaciones entre las TCEs con las medidas psicométricas de $g$.

La correlación entre $g$ y TR no se puede explicar por el balance rapidez-precisión, el uso de estrategias cognitivas o la motivación. Tampoco se debe a que los tests de CI empleados exijan un control del tiempo: la mayor parte de los estudios están basados en tests de CI resueltos por los sujetos sin un control del tiempo (p.e. el Raven). En consecuencia, la correlación entre $g$ y TR parece ser un reflejo de las diferencias individuales en la velocidad y eficiencia del procesamiento de la información. Existe un factor general de velocidad de procesamiento que es común a todas las TCEs y este factor general presenta un peso muy alto en $g$; ello sugiere que $g$ se puede explicar, al menos en parte, por la velocidad y eficiencia del procesamiento de información. Este hecho podría estar relacionado con algunas propiedades comunes a todas las regiones y módulos cerebrales que están al servicio de las funciones cognitivas que contribuyen a las diferencias individuales en población neurológicamente normal.

En suma, la memoria de trabajo se ha vinculado a menudo con la CPU de un ordenador. Esta memoria de trabajo constituye un procesador de un solo canal, de modo que cuando está actuando sobre un determinado material no puede actuar sobre otro material. Por consiguiente, cuando se presenta sucesivamente nueva información, el sujeto debe gestionar un balance entre procesamiento y almacenamiento, por lo que procesar a mayor velocidad se convierte en una ventaja. Las tareas que ponen a prueba a la memoria de trabajo constituyen un buen reflejo de las diferencias individuales en $g$.

\section{Implicaciones Para la Comprensión de las Diferencias de Inteligencia}

Cada vez más, los científicos de la conducta tratan de encontrar explicaciones cognitivas a las aptitudes. Estas, como es sabido, se basan en el análisis de las diferencias individuales observadas en las situaciones estandarizadas conocidas con el nombre de tests. Recuérdese, en este sentido, las simulaciones por ordenador de los procesos cognitivos seguidos por sujetos de alto y de medio rendimiento en los ítems del test de matrices progresivas de Raven. Pero, ¿por qué se intenta comprender las aptitudes en los términos de la ciencia cognitiva?

Resolver los problemas incluidos en los tests exige, al menos, representarse mentalmente la información de esos problemas y utilizar una serie de procesos cognitivos para manipular mentalmente esa información. La combinación de las representaciones y de los procesos produce estrategias cognitivas que los sujetos emplean para intentar resolver los problemas que se les plantean. Es un hecho obvio que algunos sujetos tienen más éxito al resolver los problemas y que otros tienen menos éxito. ¿De qué depende esa diferencia conductual? También aquí caben pocas dudas respeto a que existe alguna diferencia en las estrategias cognitivas que emplean unos y otros sujetos.

Pero, ¿en qué consiste esta diferencia? Las posibilidades que abre la ciencia cognitiva son enormes: la diferencia puede estar en los procesos atencionales, en el almacenamiento transitorio de la información en la memoria de trabajo o en el procesamiento que ahí tiene lugar, en los procesos de recuperación de información desde la memoria permanente, en la organización de la base de conocimientos de los distintos sujetos, en la cantidad de información que se puede procesar por unidad de tiempo, en el uso de determinados procesos cognitivos y un largo etcétera. A su vez, se puede intentar explicar en esos términos cognitivos las más de 60 aptitudes incluidas en modelos psicométricos como el de Carroll (1993).

Esta gran variedad de aptitudes y de posibles explicaciones cognitivas exige utilizar un método usual en la investigación científica: la simplificación. Cuando un fenómeno nos desborda, una solución viable consiste en simplificarlo. Ahora bien, ¿cómo simplificarlo del modo más inteligente posible? Albert Einstein declaró en una ocasión: "todo debe hacerse lo más simple posible, pero no más simple”. Un criterio podría ser el empírico. Así, por ejemplo, podríamos preguntarnos qué aptitud explica más información cuando 
se emplean tests de inteligencia para medir la conducta inteligente de las personas. Las evidencias revisadas en este artículo, consideradas a la luz de la investigación previa, apuntan claramente hacia $g$. En consecuencia, en un primer intento de simplificar, la primera aptitud que debería intentar someterse a consideración desde una perspectiva cognitiva sería $g$, pues $g$ es una fuente común de diferencias individuales en todos los tests estandarizados que hasta la fecha se han empleado; ninguna otra aptitud explica tanta varianza como $g$. Existen, además, numerosas evidencias prácticas que indican que $g$ es la aptitud responsable de que las medidas obtenidas con los tests se asocien de un modo tan significativo con fenómenos tan dispares como el rendimiento escolar, el rendimiento ocupacional, la salud general, la delincuencia, las ondas cerebrales, el consumo energético cerebral, los índices de heredabilidad calculados por los genéticos de la conducta, la motivación, la creatividad, el altruismo, el liderazgo, los intereses, los ingresos, la respuesta a la psicoterapia, el nivel socioeconómico alcanzado por un individuo, y un largo etcétera (Jensen, 1998; Lubinski, 2000). Es decir, el rendimiento en los tests se asocia con todos esos fenómenos en la medida en que esos tests miden $g$ : cuanto mejor miden $g$, más intensamente se asocian con esos fenómenos (Colom \& Andrés-Pueyo, 1999).

Ahora que sabemos cuál es la primera aptitud de la inteligencia, ¿cuáles de las posibilidades que nos ofrece la ciencia cognitiva se muestra especialmente relevante? También aquí parece haber pocas dudas: la velocidad de procesamiento y la memoria de trabajo, constituyen los candidatos más firmes. Se ha sugerido, en este sentido, que el cerebro consciente puede actuar como un solo canal o un sistema de procesamiento de información de capacidad limitada. Ese sistema puede transmitir simultáneamente una cantidad muy limitada de información. Las limitaciones de capacidad reducen el número de operaciones que se pueden realizar a la vez con la información que entra en el sistema. Desde esta perspectiva, resultará ventajosa la velocidad de las operaciones mentales para que se puedan realizar más operaciones por unidad de tiempo sin sobrecargar el sistema. Puesto que existe un rápido desvanecimiento de las huellas de los estímulos y de la información dentro del sistema, la velocidad de cualquier operación que pueda realizarse mientras la información está aún disponible en la memoria de trabajo es una clara ventaja. Cuanto más compleja es la información y más operaciones se requieren, más tiempo se precisa y, por consiguiente, mayor será la ventaja de la velocidad que se le pueden imprimir a los procesos elementales. La ausencia de información que se puede atribuir a una interferencia por sobrecarga y a un desvanecimiento de las huellas codificadas erróneamente o el intento de almacenamiento y recuperación de la memoria permanente, supone un fracaso por el que no se consigue comprender todas las relaciones esenciales entre los elementos de un problema complejo. En palabras de Jensen (1982):

la velocidad en el procesamiento de la información debe guardar relación con el éxito al tratar una tarea cognitiva, en la medida en que el volumen de información pone a prueba el canal de capacidad limitada del sujeto. Las tareas que diferenciarían mejor dentro de una prueba serían aquellas que amenazan el sistema de procesamiento de información llevándolo al borde del colapso. En una serie de tareas de distinta complejidad, este colapso se producirá en distintos puntos según los diferentes individuos (p. 181).

En un intento de contrastar estas relaciones entre aptitudes y parámetros cognitivos, se realizaron tres amplios estudios dentro de un proyecto de colaboración entre la Universidad Autónoma de Madrid (UAM) y el Laboratorio Armstrong de la US Air Force. Se evaluaron las aptitudes verbal, numérica y figurativa, así como los dos parámetros cognitivos ya comentados: la velocidad de procesamiento y la memoria de trabajo (Colom, Palacios, Juan-Espinosa \& Abad, 1998; Palacios, 1997).

El primer estudio se realizó en la UAM con 198 estudiantes de Psicología. El segundo estudio se llevó a cabo en el Armstrong Lab. con 203 reclutas. El tercer estudio se realizó también en el Armstrong Lab. con 193 reclutas. Por tanto, se estudió un total de 594 sujetos. Se usaron varias pruebas psicométricas y cognitivas que representaban un amplio espectro de contenido y nivel de complejidad. Respecto al contenido se usaron tareas verbales, figurativas y numéricas. Por cada contenido se seleccionaron o construyeron tareas de distinto nivel de complejidad. Las menos complejas exigían más velocidad que precisión, mientras que las más complejas exigían más precisión que velocidad.

¿Cuáles fueron los resultados de estos tres amplios estudios? Una de las principales conclusiones es que la velocidad de procesamiento y la memoria de trabajo son dos dimensiones distinguibles, aunque no independientes. La velocidad influiría positivamente en el rendimiento. Sirviéndonos de la metáfora del artista circense sugerida por Anderson (1995), el número de platos que el artista puede mantener en movimiento (memoria de trabajo) depende de modo relevante de la velocidad que les imprima (velocidad de procesamiento). Las relaciones negativas que se observaron en algunos casos entre rapidez y precisión, pueden significar que los sujetos más precisos son capaces de realizar todas las transformaciones y comparaciones exigidas por los problemas. Hay que decir que esta interpretación sería congruente con los análisis de Just y Carpenter (1992) sobre las tareas de comprensión lingüística antes comentadas. Además, la relación entre rapidez y precisión sería un índice del grado de complejidad de las tareas: (a) cuando las tareas de memoria de trabajo son poco complejas, existe una relación positiva entre velocidad y precisión. (b) cuando aumenta su complejidad, se reduce la relación entre velocidad y precisión. (c) cuando las demandas de las tareas de memoria de trabajo son verdaderamente altas, la relación entre velocidad y precisión pasa a ser negativa. Los resultados (b) y (c) podrían ser explicados porque la resolución del ítem exige un tiempo superior a 1 segundo y medio, facilitando, de este modo, la participación de procesos cognitivos de alto nivel que harían irregulares las relaciones observadas entre velocidad y precisión (Jensen, 1998; Palacios, 1997). 
¿Cuál es la relación entre el contenido de las tareas (verbal, figurativo y numérico) y su nivel de complejidad? En las pruebas psicométricas, el contenido es relevante en algunas tareas: (a) las pruebas psicométricas de velocidad se sitúan en el mismo punto que las pruebas cognitivas de velocidad; (b) las pruebas psicométricas algo más complejas se sitúan en un punto de importancia relevante del contenido; (c) las pruebas psicométricas más complejas se sitúan en un punto de mucha importancia del contenido. Por lo que se refiere a las pruebas cognitivas, se observó que el contenido es poco relevante, tanto para las tareas de velocidad de procesamiento como para las tareas de memoria de trabajo. Sin embargo, en estos tres estudios no se exploró un rango representativo de complejidad en el caso de las pruebas cognitivas.

En consecuencia, los resultados de esos tres estudios no permiten sugerir que la velocidad con la que se procesa la información y la memoria de trabajo son independientes, un resultado congruente con las propuestas conceptuales derivadas del estudio con TCEs. En las tareas que exigen esencialmente precisión (caso de las tareas de memoria de trabajo) la velocidad con la que el sujeto procesa la información puede influir positiva o negativamente: el sujeto más preciso será también el más lento en las tareas más complejas, pero el sujeto más preciso será también el más rápido en las tareas más simples. Por lo que se refiere a las tareas de mediana complejidad, la relación entre precisión y velocidad será moderada. En cuanto a las relaciones entre nivel de complejidad y contenido de la información a procesar, parece que solamente en las tareas de mediana complejidad se observará una relevancia significativa del contenido respecto a la actuación del sujeto. En cambio, tanto en las tareas poco complejas como en las muy complejas, el contenido de las tareas contribuirá poco a explicar el rendimiento del sujeto: un sujeto que tenga un alto rendimiento en una prueba muy compleja o muy sencilla lo seguirá teniendo aunque se le exija procesar distintos tipos de información (contenidos), pero esto no tendrá porque ser así en tareas de mediana complejidad (donde el contenido de la información será relevante). Finalmente, las restricciones de la capacidad general del sujeto serán tanto más relevantes cuanto más complejas sean las tareas que deba resolver: las restricciones de capacidad jugarán un papel relativamente escaso en las tareas de baja complejidad, pero el incremento gradual de la complejidad se verá reflejado en una reducción del rendimiento de los sujetos de menor capacidad. Esto último es consistente con la tesis y los resultados del equipo del Carnegie Mellon (Just \& Carpenter, 1992).

En suma, las diferencias individuales en la inteligencia evaluada a través de los tests estandarizados se asocian con muchos fenómenos con repercusiones sociales. El principal responsable de esa asociación es $g$. Las diferencias individuales en $g$ parecen estar asociadas a conceptos cognitivos como la velocidad de procesamiento y la memoria de trabajo. Desde esta perspectiva, las diferencias individuales en $g$ serían debidas a las diferencias individuales observadas en la capacidad de la memoria de trabajo, es decir, la capacidad del sujeto para manipular simultáneamente la información necesaria para intentar resolver un problema cognitivamente exigente. Una mayor velocidad para procesar esa información confiere una ventaja al sujeto, contribuyendo a superar los puntos de colapso del sistema de procesamiento de información.

En la historia de la psicología se han realizado muchos proyectos destinados a mejorar la inteligencia, puesto que se trata de un fenómeno psicológico de indudable trascendencia social. Esos programas han intentado mejorar la inteligencia precisamente debido a la intensidad con la que la inteligencia se asocia con muchos fenómenos sociales; se suponía que mejorar la inteligencia produciría una mejora en los fenómenos con los que se asocia. Sin embargo, los intentos han tenido menos éxito del esperado (Neisser y cols., 1996). Quizá ahora estamos en disposición de saber por qué: si, en efecto, las diferencias de inteligencia dependen de la velocidad de procesamiento y de la capacidad de la memoria de trabajo, entonces los intentos de mejorar la inteligencia deberían ir destinados a manipular los puntos de colapso que los sujetos padecen por efecto de las limitaciones de capacidad de esa memoria de trabajo. Hallemos los modos de superar esas limitaciones de capacidad y habremos encontrado quizá el modo de mejorar la inteligencia.

\section{Referencias}

Anderson, J. (1995). Rules of the mind. New Jersey:LEA.

Andrés Pueyo, A. (1997). Manual de psicología diferencial. Barcelona: McGraw-Hill.

Baddeley, A. (1986). Working memory. London: Oxford Univ. Press. Baddeley, A. (1997). Human Memory. Theory and Practice. New York: Mc Graw Hill.

Baddeley, A., Lewis, V. \& Vallar, G. (1984). Exploring the Articulatory Loop. The Quarterly Journal of Experimental Psychology, 36A (2). 233-252.

Baddeley, A. \& Wilson, B. (1985). Phonological Coding and ShortTerm Memory in patients without Speech. Journal of Memory and Language, 24, 490-502.

Carpenter, P. \& Just, M. (1988). The role of working memory in language comprehension. En D. Klahr \& K Kotovsky (Orgs.), Complex information processing: the impact of Herbert Simon. Hillsdale, NJ: LEA.

Carpenter, P.A., Just, M.A. \& Shell, P. (1990). What one intelligence test measures: a theoretical account of the processing in the raven progressive matrices test. Psychological Review, 97, 404-431.

Carroll, J.B. (1993). Human cognitive abilities. A survey of factor analytic studies. Cambridge : Cambridge Univ. Press.

Carroll, J.B. (1997). Psychometrics, intelligence, and public perception. Intelligence, 24, 25-52.

Colom, R. (1998). Psicología de las diferencias individuales. Teoría y práctica. Madrid: Pirámide.

Colom, R. \& Andrés-Pueyo, A. (1999). El estudio de la inteligencia humana: recapitulación ante el cambio de milenio. Psicothema, 11, 453-476.

Colom, R. \& Flores-Mendoza, C. E. (enviado). Working memory and fluid intelligence. Memory \& Cognition. 
Colom, R., Juan-Espinosa, M., Abad, F. \& Garcia, L.F. (2000). Negligible sex differences in general intelligence. Intelligence, 28, 57-68.

Colom, R., Palacios, R., Kyllonen, P. \& Juan-Espinosa, M. (enviado). Working memory is not distinguishable from $g$. Intelligence.

Colom, R., Palacios, A., Juan-Espinosa, M. \& Abad, F. (1998). Inteligencia y memoria de trabajo. En $\mathrm{M}^{\mathrm{a}}$ Pilar Sánchez-López y $\mathrm{M}^{\mathrm{a}}$ Angeles Quiroga Estevez (Orgs.), Perspectivas actuales en la investigación psicológica de las diferencias individuales. Madrid: Fundación Ramón Areces.

Dempster, F. (1992). The rise and fall of the inhibitory mechanism: toward a unified theory of development and aging. Developmental review, 12, 45-75.

Estes, W.K. (1987). One Hundred Years of Memory Theory. En: Gorfein, D.S. y Hoffman, R.R (Orgs.), Memory and Learning. The Ebbinghaus Centennial Conference. New Yersey:Lawrence Erlbaum Associates, Publishers.

Flores-Mendoza, C.E. (1999). Processamento cognitivo básico e inteligência em deficientes mentais. Tese de Doutorado, Universidade de São Paulo.

Flores-Mendoza, C.E. \& Colom, R. (en prensa). Memoria de trabajo, retraso mental, y dificultades de aprendizaje. Estudos de Psicología.

Hagendorf, H. \& Sá, B. (1995). Coordination in visual working memory. Zeitschrift für psychologie, 203, 53-72.

Hunt, E.B. (1971). What kind of computer is man? Cognitive Psychology, 2, 57-98.

Hunt, E.B., Lunneborg, C. \& Lewis, J. (1975). What does it mean to be high verbal? Cognitive Psychology, 7, 194-227.

Jensen, A. (1982). Reaction time and psychometric $g$. En: H.J. Eysenck (Org.): A model for intelligence. Berlín: Springer Verlag.

Jensen, A. (1993). Spearman's $g$ : links between psychometrics and biology. Brain Mechanisms, 702, 103-129.

Jensen, A. (1998). The g factor. New York: Praeger.

Jensen, A. \& Weng, L. (1994). What is a good $g$ ? Intelligence, 18, 231-258.

Juan-Espinosa, M. (1997). Geografía de la inteligencia humana. Madrid: Pirámide.

Just, M. \& Carpenter, P. (1992). A capacity theory of comprehension: individual differences in working memory. Psychological Review, 99, 122-149.
Just, M., Carpenter, P. \& Keller, T. (1996). The theory of comprehension: new frontiers of evidence and arguments. Psychological Review, 103, 773-780.

Kyllonen, P. \& Christal, R. (1990). Reasoning ability is (little more than) working memory capacity?! Intelligence, 14, 389-433.

Logie, R. (1996). The seven ages of working memory. En J.T.E. Richardson (Org.): Working memory and human cognition. Oxford: Oxford Univ. Press.

Lubinski, D. (2000). Scientific and social significance of assessing individual differences: "Sinking shafts at a few critical points". Annual Review of Psychology, 51, 405-444.

Neisser, U., Boodoo, G., Bouchard, T., Boykin, A., Brody, N., Ceci, S., Halpern, D., Loehlin, J., Perloff, R., Sternberg, R. \& Urbina, S. (1996). Intelligence: Knows and Unknows. American Psychologist, 51, 77-101.

Oberauer, K. (1993). The coordinarion of cognitive operations. A study on the relationship of working memory and intelligence. Zeitschrift für psychologie, 201.

Oberauer, K., Süb, H., Schulze, R., Wilhelm, O \& Wittmann. W. (2000). Working memory capacity -facets of a cognitive ability construct. Personality and Individual Differences, 29, 10171046.

Morrin, K., Law, D. \& Pellegrino, J. (1994). Structural modeling of information coordination abilities: an evaluation and extension of the Yee, Hunt and Pellegrino model. Intelligence, 19, 117-144.

Palacios, A. (1997). Inteligencia psicométrica e inteligencia cognoscitiva. Estudio de las demandas de velocidad y complejidad de procesamiento. Tesis Doctorado. Universidad Autónoma de Madrid.

Salthouse, T. (1990). Working memory as a processing resource in cognitive aging. Developmental review, 10, 101-124.

Smith, A.D. (1996). Memory. En J.E. Birren \& K.W. Schaie (Orgs.), Handbook of psychology and aging. $4^{a}$ Edition. Nueva York: Academic Press.

Smith, E.E. \& Jonides, J. (1997). Working memory: a view from neuroimaging. Cognitive psychology, 33, 5-42.

TEA (1998). Manual del WAIS III. Madrid: TEA.

Waters, G. \& Caplan, D. (1996). The capacity theory of sentence comprehension: critique of Just and Carpenter (1992). Psychological Review, 103, 761-772. 